\title{
Evaluation of Low-Intensity Extracorporeal Shock Wave in the Treatment of Peyronie's Disease: A Single Arm Observational Study
}

\author{
(D) Ibrahim Nüvit Tahtalı ${ }^{1}$, (1) Turgay Karataş ${ }^{2}$ \\ ${ }^{1}$ Department of Urology, Malatya Training and Research Hospital, Malatya, Turkey \\ ${ }^{2}$ Department of Anatomy, İnönü University, Malatya, Turkey
}

\begin{abstract}
Introduction: The purpose of this study was to determine the efficacy of low-intensity extracorporeal wave therapy (LiESWT) in the treatment of Peyronie's disease (PD).

Methods: Li-ESWT was used to treat 41 patients applied with PD using the Duolith SD1 UItra device. In Peyronie's patients, a total of 300 beats $\left(3,000\right.$ strokes $\left.0.25 \mathrm{~mJ} / \mathrm{mm}^{2}\right)$ were applied to the penile region to be Li-ESWT with clinical emphasis, that is, on the palpable Peyronie's plaque, once a week for 6 weeks. The method's reproducibility and efficacy were determined by comparing penile discomfort, penile curvature, penile plaque size, and International Index of Erectile Function (IIEF-5) score values in Peyronie's patients at baseline and 3 months, 6 months, and 1 year after Li-ESWT treatment.

Results: While there was a statistically significant decrease $(p<0.001)$ in Vas score before and after Li-ESWT treatment at 3, 6, and 12 months, the change in angulation in IIEF-5 scoring and plaque size was not significant $(p=0.950, p=0.162, p=0.162$, respectively).

Discussion and Conclusion: These results showed us that Li-ESWT treatment in PD did not provide angulation, shrinkage of penile plaque and improvement in sexual functions, except for reducing the penile pain complaint of the patients during erection. Findings indicate that Li-ESWT alleviates penile pain associated with PD. However, it did not appear to be useful in terms of reducing penile curvature, plaque size, or sexual function.

Keywords: Extracorporeal shock wave therapy; penis; peyronie's disease.
\end{abstract}

$P$ eyronie's Disease (PD), identified for the 1st time in 1743 by French surgeon Francois Gigot de La Peyronie, ${ }^{[1]}$ is characterized as fibrotic alteration of the penis with localized plaques, penile deformities, shortening, pain, and erectile dysfunction (ED) in the tunica albuginea. Between $0.4 \%$ and $13 \%$ of males between the ages of 40 and 70 have been reported to have PD ${ }^{[2]}$.

Although the cause of PD is uncertain, it is caused mainly by recurrent mild trauma episodes that damage microvascular structures and promote fibroblast proliferation in the tunica albuginea. The differentiation of these fibroblasts into myofibroblasts can result in excessive collagen deposition, fibrin persistence, and elastin breakdown, eventually resulting in connective tissue remodeling, fibrotic plaque development, and aberrant penis curvature ${ }^{[3]}$.

Its incidence increases with age. The most noticeable fea-

Correspondence (İletişim): İbrahim Nüvit Tahtalı, M.D. Malatya Egitim ve Arastirma Hastanesi, Malatya, Turkey

Phone (Telefon): +90 5326112182 E-mail (E-posta): nuvit_tahtali@hotmail.com

Submitted Date (Başvuru Tarihi): 15.12.2021 Accepted Date (Kabul Tarihi): 04.01.2022

Copyright 2022 Haydarpaşa Numune Medical Journal

OPEN ACCESS This is an open access article under the CC BY-NC license (http://creativecommons.org/licenses/by-nc/4.0/) 
ture is penile deviation, narrowing, and fibrous plaques, which might cause distal instability. Dorsal curvature is the most frequent type of deviation, followed by ventral and lateral curvature. It has been reported that pain is usually always a self-limiting condition ${ }^{[4]}$.

There are two phases defined in PD: acute and chronic phases. The acute phase is characterized by a painful perivascular inflammatory reaction during erection and lasts 2-10 months. In the second stage, the chronic phase, progressive fibrosis with collagen and fibrinous tissue is observed with the formation of palpable plaques. This characteristic plaque causes curvature deformity of the erect penis ${ }^{[5]}$.

Numerous treatment modalities have been reported for $\mathrm{PD}$, both conservative and invasive. If curvature correction is the primary objective of PD treatment, surgery is the gold standard. It is advised for individuals whose disease has been stable for at least 3 months before surgery [6]. Oral, intralesional, and topical therapies are considered conservative therapy for Peyroni's treatment ${ }^{[7]}$.

Nearly 40 years ago, medicine launched extracorporeal shock wave treatment (ESWT). The mechanism by which shock waves (SW) influence biological creatures is unknown. Contrary to lithotripsy, which has a damaging impact on urinary stones, it has been claimed that SW has restorative potential in various organs ${ }^{[8]}$.

This study aimed to determine the effect of low-intensity ESWT (Li-ESWT) on the symptoms and sexual functioning of PD.

\section{Materials and Methods}

\section{Ethical Status}

The Malatya clinical research ethics committee, accepted this study (Date: 09.12.2020, Decision no: 2020/182). The study adhered to the International Helsinki Declaration and other relevant statements. Between July 2017 and May 2018, 41 PD patients were treated with Li-ESWT at Malatya Divan Hospital's urology department. Each patient received extensive information on the available treatment choices for PD. The age, length of complaint, location of the penile plaque, responsiveness to treatment, and post-procedural problems of patients were retrospectively examined.

As advised in the literature, the effectiveness of Li-ESWT was determined by analyzing penile deviation/curvature, plaque size, ED, and penile pain [3].

The degree of curvature of the penis was measured and recorded from three angles (front, side, and above) using images taken before and after treatment when the patient's penis was a full erection. In patients who could not obtain a sufficient erection, $20 \mathrm{mg}$ alprostadil (Pharmacia and Upjohn, Puurs, Belgium) was given intracavernally to induce an erection. In our study, four patients required intracavernous injections of alprostadil.

The dimensions of the Peyronie's plaque in square centimeters were measured by multiplying the plaque's breadth and length perceptible on the penis. The International Index of Erectile Function (IIEF-5) questionnaire was used to assess the patients' erectile function in this study [9].

The Visual Analogue Scale (VAS) was used to assess the degree of uncomfortable erections. VAS scoring is used for digitizing some values that are not numerically quantifiable. At the two ends of a $100 \mathrm{~mm}$ line, two end definitions of the parameter to be evaluated are printed, and the patient is asked to identify where on this line their condition is acceptable by drawing a line, inserting a dot, or pointing. At the end, when it comes to pain, he has no pain on one end, and extreme pain on the other, and the patient indicates his or her current status on this line. It was scored on a scale of $0-10$, with 0 representing no pain and 10 representing extreme pain in this scoring system.

The following criteria were used to choose patients: The study included patients with PD over 18 years, had penile plaque and/or pain during erection, and had disease symptoms lasting more than 12 months. The fact that the patients had already received medical treatment did not preclude their treatment in the study.

Patients with prior penile surgery, complex curvature (Plan Peyronie's patients with an angulation greater than 90 degrees), patients with ED who did not respond to medical treatment with Phosphodiesterase-5, patients with malignant disease, patients with coagulation disorders, patients with active infection, and patients with cardiac pacemakers were excluded from this study.

\section{ESWT Technic}

All patients were started on an ESWT device (Duolith ${ }^{\circledR}$ SD1 ultra; Storz medical ag, Tägerwilen, Switzerland) with a low energy flow density of $0.03 \mathrm{~mJ} / \mathrm{mm}^{2}$, and the power was gradually increased to the patient's tolerance level, with SW typically reaching $0.25 \mathrm{~mJ} / \mathrm{mm}^{2}$ perpendicular to the penile shaft.

In Peyronie's patients, the penile region to be ESWT was applied with a clinical focus, that is, on the palpable Peyronie's plate. Each patient was treated with six sessions, once a week for 6 weeks, with 3,000 strokes at $0.25 \mathrm{~mJ} / \mathrm{mm}^{2}$ in 
each session. This process took about 15 min per session. The same operator administered the treatments without using anesthesia or analgesics.

The treatment's adverse effects were documented. During the 6-week treatment period and the 12-month follow-up, patients were requested not to get any additional treatment for ED or PD.

\section{Statistical Analysis}

SPSS v25 was used for all analyses (SPSS Inc, Chicago, IL, USA). The Kolmogorov-Smirnov test was used to determine the normality of numerical variable variables. The Friedman test was performed to compare the variables not normally distributed among the four groups. The Wilcoxon Signed Rank Test was used to compare repeated measures of continuous variables between the two groups. In addition, the Repeated Measures Anova Test was employed to compare the four groups' customarily distributed variables. Statistical significance was defined as $p<0.05$.

\section{Results}

The patients in this retrospective single-blind randomized study ranged from 26 to 74 (median 57). Prior to the patients in the study applying to us, the average duration of PD was 6-24 months (mean, 13 months). Patients' penile examinations were used to detect plaques. Table 1 summarizes the distribution and frequency of these plaques.

While there was a statistically significant drop in VAS scoring $(p<0.001)$ between pre- and post-ESWT treatment at 3 months, 6 months, and 12 months, the change in angulation was not significant in IIEF-5 scoring or plaque size $(p=0.950, p=0.162$, and $p=0.162$, respectively). The entire set of results is depicted in Figure 1.

The pain experienced by patients during the application does not require analgesic treatment and does not persist beyond the ESWT session.

Penile petechiae were observed in four patients 2 days after the ESWT session and healed within 2 days without the need for further treatment $(9.75 \%)$. After the third session,

Table 1. Peyronie's plaque locations and rates in the penis

\begin{tabular}{lcc}
\hline Plaque localization & Number of Patients & $\%$ \\
\hline Dorsal & 21 & 48.78 \\
Ventral & 6 & 14.63 \\
Hourglass & 3 & 14.63 \\
Right Lateral & 5 & 12.20 \\
Left Lateral & 6 & 14.63 \\
\hline
\end{tabular}

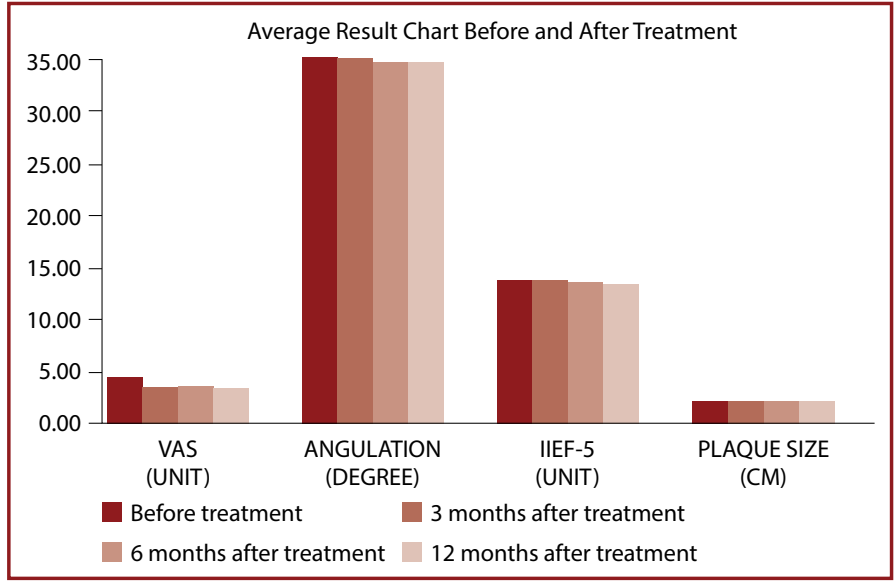

Figure 1. Average results chart before and after treatment.

one patient (2.5\%) developed a $2 \mathrm{~cm}$ ecchymosis, which healed spontaneously within 3 days. Hematuria was not observed in any of our patients, even though it is reported infrequently in the literature.

\section{Discussion}

Numerous treatments have been attempted to treat PD, including surgery (oral, intralesional, and topical therapy) and conservative treatments. While collagenase and intralesional therapy can produce satisfactory outcomes, the therapeutic effects of conservative treatments are unknown. In addition, while surgery is considered one of the most effective treatments for PD, it might cause iatrogenic penile shortening and ED. Li-ESWT was initially utilized as a topical treatment for Peyroni's disease in 1989. Since LiESWT was identified as a potentially beneficial new treatment option, several studies have evaluated its efficacy and safety. However, several highly contentious findings have been reported ${ }^{[10]}$.

In the 1980s, ESWT was defined as "mechanotherapy" with the original applications for lithotripsy in the treatment of urinary tract stones. More recently, it has also been successfully applied to regenerative medicine. The molecular mechanisms of Li-ESWT are associated with different pathways of biological reactions through a "mechano-transduction" process. Extensive basic science research has revealed that Li-ESWT does not cause mechanical disruption in tissues and cells, but instead induces biological effects that activate a series of cellular events responsible for the therapeutic effects of Li-ESWT [11].

Numerous cells, such as stem cells (SCs), bone marrow stromal cells, osteoblasts, endothelial cells, and Schwann cells, are possible targets for Li-ESWT-mediated mechanotransduction. $70-80 \%$ of SCs in the penis are concentrated in the 
subtunical area. These SCs are reactivated and cause angiogenesis when Li-ESWT is applied ${ }^{[12]}$.

Two methods are postulated for Li-ESWT to activate the PD environment. First, it is thought that the SW directly disturb the PD plaque, resulting in its destruction via an inflammatory reaction. Second, SW are thought to induce angiogenesis surrounding plaques, resulting in plaque resorption $[3,13]$. After it was demonstrated that Li-ESWT activates local progenitor cells, it was reported that it might be utilized in exogenous SC therapy, an invasive treatment used to activate ED [13].

The angiogenic effects of Li-ESWT treatment are thought to be due to the mechanical damage caused by the waves. This induces the synthesis of angiogenic growth factors such as vascular endothelial growth factor and endothelial nitric oxide synthase, promoting neovascularization ${ }^{[12,14]}$.

Palmier et al., [15] reported the first placebo-controlled randomized study examining the effect of Li-ESWT on Peyroni's disease. The study enrolled 100 patients with a PD history lasting $<12$ months. The results indicated a statistically significant improvement in pain, quality of life (QoL), and IIEF-5 score between the two groups and between weeks 12 and 24. Compared to the placebo arm, $83 \%$ of patients in the ESWT group reported relief in pain, compared to $43 \%$ in the control arm. IIEF-5 rose to five points more in the ESWT group than in the control group over the follow-up period. The two groups did not differ substantially in terms of penile curvature. These authors concluded that Li-ESWT alleviates pain and enhances the QoL and erectile function. Palmieri et al.,, ${ }^{[15]}$ reported in 2012 that supplementing Li-ESWT with $5 \mathrm{mg}$ of tadalafil daily boosted the therapeutic effect of the treatment in men with PD and ED. No statistically significant improvement in symptoms other than the pain was found in our study.

Kriger et al., ${ }^{[3]}$ concluded in their analysis of the effects of Li-ESWT on PD that this very noninvasive and low-risk treatment approach had a negligible effect on penile curvature plaque size erectile function. In their meta-analysis study, Gao et al., ${ }^{[10]}$ reported that while most patients may have disease progression or stability during the disease, $89 \%$ of men will have entirely alleviated pain symptoms within 18 months of disease beginning. According to these researchers, the effect of Li-ESWT on symptoms other than penile pain is highly questionable, and it imposes an extra expensive burden on patients $[3,10,16]$. While the change in Vas scoring was statistically significant $(p<0.001)$ before and after ESWT treatment at 3 months, 6 months, and 12 months, no significant improvement in angulation, IIEF-5 scoring, or plaque size was observed ( $p>0.05$ ).

There are few risks associated with the use of shockwave therapy in PD and most adverse side effects are very minor in nature. Following treatment, local pain during the application, skin hematoma, and local petechiae are the most frequently reported side effects, all of which resolve spontaneously [1]. In our study, we observed penile petechiae in four patients (10.25\%) and penile ecchymosis in one patient (2.4\%). Penile petechiae and penile ecchymosis disappeared spontaneously within 2 days and 3 days, respectively. Hematuria resulting from urethral hemorrhage has been reported seldom ${ }^{[10]}$. Krieger et al., ${ }^{[3]}$ reported an increase in penile curvature in patients with PD following Li-ESWT treatment. However, no other study has corroborated this. By and large, it looks as though this kind of treatment is pretty safe for the patient.

Penile pain improves more rapidly in PD patients treated with Li-ESWT than it does in the disease's natural course. However, the question arises as to whether pain should be treated at all, given that most patients would heal spontaneously over time. Eighty-nine percent of patients with PD will experience no pain after an average of 18 months without treatment. Pain is most frequently experienced during the acute phase, which lasts around 12-18 months ${ }^{[17]}$. The potential value of ESWT in terms of pain reduction is exaggerated, given the treatment's requirement of at least six sessions and the associated cost load on the patient and healthcare system. However, patients whose pain has a significant impact on their lives (i.e., those who require frequent analgesics or refrain from sexual activity due to penile pain) may be suitable candidates for ESWT provided they are informed that the treatment is intended to alleviate pain alone ${ }^{[17,18]}$.

\section{Limitations}

The study's primary weaknesses are its lack of a control group, retrospective design, and failure to assess the effects of Li-ESWT delivered at various dosages during the study.

\section{Conclusion}

Li-ESWT appears to be a straightforward way of treating PD without significant problems; however, its efficacy is highly debatable. According to the findings of this retrospective study, Li-ESWT did not affect Peyronie's plaque size, penile curvature, or sexual dysfunction. However, it was concluded that penile pain caused by PD disappeared more rapidly following Li-ESWT treatment than through- 
out the illness's natural course. When evaluated with other prior research, it was concluded that Li-ESWT should not be suggested as a routine treatment for Peyroni's disease. However, more significant controlled trials are necessary to evaluate Li-efficacy ESWT's in treating PD.

Ethics Committee Approval: This study was approved by the Malatya clinical research ethics committee (Date: 09/12/2020, decision no: 2020/182). The study was carried out following the International Helsinki and other declarations.

Peer-review: Externally peer-reviewed.

Authorship Contributions: Concept: I.N.T.; Design: I.N.T.; Data Collection or Processing: I.N.T.; Analysis or Interpretation: I.N.T.; Literature Search: T.K.; Writing: I.N.T.

Conflict of Interest: None declared.

Financial Disclosure: The authors declared that this study received no financial support.

\section{References}

1. Ahmadnia $H$, Kamalati $A$, Younesi Rostami M, Imani MM, Asadpour AA, Hariri MK. The therapeutic effects of intracavernosal plaque excision in peyronie's disease: A none grafting or tunical excising procedure. World J Plast Surg 2016;5:62-6.

2. Salabas E, Ozmez A, Ermec B, Cevik G, Akdere H, Kadioglu A. Penile curvature after Peyronie's disease surgery: What are the risk factors? Andrologia 2020;52:e13860. [CrossRef]

3. Krieger JR, Rizk PJ, Kohn TP, Pastuszak A. Shockwave therapy in the treatment of Peyronie's disease. Sex Med Rev 2019;7:499507. [CrossRef]

4. Fojecki GL, Tiessen S, Osther PJ. Extracorporeal shock wave therapy (ESWT) in urology: A systematic review of outcome in Peyronie's disease, erectile dysfunction and chronic pelvic pain. World J Urol 2017;35:1-9. [CrossRef]

5. Brimley SC, Yafi FA, Greenberg J, Hellstrom WJG, Tue Nguyen HM, Hatzichristodoulou G. Review of management options for active-phase Peyronie's disease. Sex Med Rev 2019;7:32937. [CrossRef]

6. Talib RA, Ibrahim MA, Cangüven Ö. Nonsurgical treatment options in Peyronie's disease: 2016 update. Turk J Urol 2016;42:217-23. [CrossRef]

7. Russo Gl, Milenkovic U, Hellstrom W, Levine LA, Ralph D, Albersen M. Clinical efficacy of injection and mechanical therapy for Peyronie's disease: A systematic review of the literature.
Eur Urol 2018;74:767-81. [CrossRef]

8. Porst $\mathrm{H}$. Review of the current status of low intensity extracorporeal shockwave therapy (Li-ESWT) in Erectile Dysfunction (ED), Peyronie's disease (PD), and sexual rehabilitation after radical prostatectomy with special focus on technical aspects of the different marketed ESWT devices including personal experiences in 350 patients. Sex Med Rev 2021;9:93-122. [CrossRef]

9. Neijenhuijs KI, Holtmaat K, Aaronson NK, Holzner B, Terwee $C B$, Cuijpers $P$, et al. The international index of erectile function (IIEF)-a systematic review of measurement properties. J Sex Med 2019;16:1078-91. [CrossRef]

10. Gao L, Qian S, Tang Z, Li J, Yuan J. A meta-analysis of extracorporeal shock wave therapy for Peyronie's disease. Int J Impot Res 2016;28:161-6. [CrossRef]

11. d'Agostino MC, Craig K, Tibalt E, Respizzi S. Shock wave as biological therapeutic tool: From mechanical stimulation to recovery and healing, through mechanotransduction. Int J Surg 2015;24:147-53. [CrossRef]

12. Lin G, Reed-Maldonado AB, Wang B, Lee YC, Zhou J, Lu Z, et al. In situ activation of penile progenitor cells with low-intensity extracorporeal shockwave therapy. J Sex Med 2017;14:493501. [CrossRef]

13. Walker DT, Amighi A, Mills SA, Eleswarapu SV, Mills JN. Managing the acute phase of Peyronie's disease: A contemporary review: Current Sexual Health Reports. 2019;22(11):370-80. [CrossRef]

14. Fode $M$, Østergren PB. Contemporary thoughts on the role of shockwave therapy in Peyronie's disease. J Sex Med 2020;17:174-9. [CrossRef]

15. Palmieri A, Imbimbo C, Creta M, Verze P, Fusco F, Mirone V. Tadalafil once daily and extracorporeal shock wave therapy in the management of patients with Peyronie's disease and erectile dysfunction: Results from a prospective randomized trial. Int J Androl 2012;35:190-5. [CrossRef]

16. Palmieri A, Imbimbo $C$, Longo N, Fusco F, Verze $P$, Mangiapia $\mathrm{F}$, et al. A first prospective, randomized, double-blind, placebo-controlled clinical trial evaluating extracorporeal shock wave therapy for the treatment of Peyronie's disease. Eur Urol 2009;56:363-9. [CrossRef]

17. Hatzichristodoulou G, Meisner C, Gschwend JE, Stenzl A, Lahme S. Extracorporeal shock wave therapy in Peyronie's disease: Results of a placebo-controlled, prospective, randomized, single-blind study. J Sex Med 2013;10:2815-21. [CrossRef]

18. Di Maida F, Cito G, Lambertini L, Valastro F, Morelli G, Mari A, et al. The natural history of Peyronie's disease. World J Mens Health 2021;39:399-405. [CrossRef] 